\title{
Enhancing college students' global awareness through campus Toastmasters clubs
}

\author{
Hsu, Tsu-Chia $\bowtie$ \\ Lunghwa University of Science and Technology, Taiwan, ROC (hsuinuk@gmail.com)
}

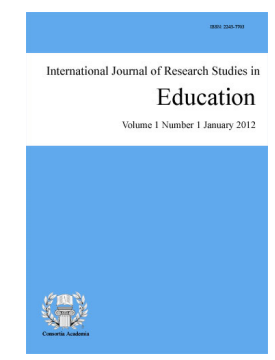

ISSN: $2243-7703$ Online ISSN: 2243-7711

OPEN ACCESS

\section{Abstract}

The main objective of this study is to investigate the effects of the 20 college campus Toastmasters clubs all over Taiwan, towards the enhancement of its student members' global awareness. Within the concept of cooperative learning, promotion of global views by means of sharing various cultural concepts within the conducted Toastmasters meeting program, group works such as structured interaction and communication activities, and individual speaking activities, while not worrying about their grammatical errors are used as strategies. Using the qualitative research paradigm in terms of focus group and individual interviews, the current study gathers college student members' global viewpoints along with their insightful observations in order to understand the implications of such cooperative strategies. Results indicates that co-curricular programs in existing campus activities, such as Campus Toastmasters clubs, is capable of developing the students' global viewpoints and/or was able to help diminish their culture shock within a competitive diversely membered higher education milieu.

Keywords: globalization; internationalization of higher education; Toastmasters; culture shock; effective communication skills; global perspective 


\section{Enhancing college students' global awareness through campus Toastmasters clubs}

\section{Introduction}

In the twenty-first century, the trend of globalization has sped up both the growth and development of higher education, particularly in most higher education institutions committed to internationalization. According to OECD (2006), since 1995 the total number of international students have doubled to a huge figure of 2.7 million students. Correspondingly the demand for international wide education is estimated to increase from 1.8 million international students in 2000 to 7.2 million in 2025 (Bohm, Meares, \& Pearce, 2002). More importantly, analysts predict that 70 percent of the global demand will be generated within the Asia Pacific region (Olsen, 2003).

In Taiwan, the trend of globalization and internationalization particularly echoes traditional East Asian patterns; wherein a great number of university students from Taiwan mainly choose to study in the United States (US) and United Kingdom (UK). However, recent efforts of the Ministry of Education (MOE) to increase the number of incoming international students have seen to be taking its effect. Ko (2008) in a speech mentioned that the total number of international students in Taiwan for 2007, including degree-level, exchange, and language study students, have reached a total of 17,742, which registers an increase of 3,263, compared to the 14,479 count in 2006. Comparing to the earlier data from the year of 2001 to 2005, incoming international student enrollments from Central and South America increased 208 percent while 95 percent from European nations. Among those students, the largest group comes from Vietnam, followed by Malaysia, Indonesia, Japan and the United States (Ko, 2008). In essence, the growing number of students studying in Taiwan is seen as positive factor in further enhancing the higher education institutions' internationalization atmosphere (Chin \& Ching, 2009).

In an internationalized campus, it is expected that foreign (international) students act as both the liaison and communicator to bridge the gap among different ethnicity and cultural background. Hence, higher education institutions in Taiwan utilizes co-curricular activities such as the Campus Toastmasters Clubs; which are co-curricular activities with a sole purpose of helping their student members gain personal growth through the training of communication and leadership skills in a positive and cooperative environment (Toastmasters International District 67, 2009a), to help enhanced the international atmosphere. In particular, these international students have taken great opportunities to exposed themselves and gain intercultural experience within a cultural exchange study abroad paradigm through cooperative learning processes such as face-to-face interaction and positive inter-dependency. Furthermore, such avenues for international cultural exchange; which the benefit is not only to diminish their cultural shock through bridging the barrier between languages and culture itself, but would also provide additional opportunities in gaining global perspective through effective communication processes.

Looking beyond language learning, good communication can inspire students to learn and participate more in class (Noels, Clément, \& Pelletier, 1999). Hence, good communication skills have become a must for college graduates (Coldstream, 1997). Furthermore, in the current globalizing age, having a broad global perspective is likewise considered as a prerequisite for future graduates in becoming a more capable person. In essence, such campus Toastmasters clubs are said to provide their members, including local and internationals students, more opportunities to exchange their global viewpoints through different thematic weekly meeting sessions.

In light of the above issues, the current study aims to investigate the effects of the twenty (20) college campus Toastmasters clubs all over Taiwan, towards the enhancement of its student members' global awareness. Furthermore, this study also seeks to understand the difficulties and barriers in the recruitment of international student members. It is hoped that this study will be able to help campus Toastmasters club members to know 
who they are and realize what they are capable of doing. In essence, helps build up their confidence with respect to effective communication and leadership skills.

\subsection{Research questions}

This study uses the qualitative research paradigm in terms of focus group and individual interviews, to gather the college student members' global viewpoints along with their insightful observations in order to understand the implications of cooperative strategies. More specific research questions are as follows:

1. How can campus Toastmasters enhance the college students' global perspective?

2. How can campus Toastmasters increase its diversity on its membership in order to gain more global point of views?

\section{Literature reviews}

In order to fully understand the concept behind this study, various background literature reviews shall be provided, namely: the definition of globalization and internationalization in higher education, and the campus Toastmasters club and its contributions.

\subsection{Definition of Globalization and Internationalization in higher education}

In the current globalizing age, most higher education institutions in Taiwan have recently shifted their focus towards becoming globally competitive. Within a wider context, globalization can be defined as the closer integration of the countries and peoples of the world, brought about by the enormous reduction of costs of transportation and communication, and the breaking down of artificial barriers to the flows of goods, services, capital, knowledge, and people across borders (Stiglitz, 2002). It also refers to the process and consequences of instantaneous communication and technological advancement, which brought about the tremendous growth in the quantity of information and integration (Grunzweig \& Rinehart, 2002). While academic systems and institutions may make different accommodations to these trends, however neither one can ignore its impetus and implications.

On the other hand, internationalization represents university policy, initiatives, and practices that are adopted in response to the affects of globalization (Scott, 1998). Parmenter (2000) echoes that internationalization "as a practice or process is a more localized phenomenon, and is not necessarily dependent on the existence of internationalization as a policy" (p.310). Within the global environment, internationalization may be divided into three perspectives: necessary knowledge of other countries, knowledge of international and global issues, and competencies including language, cultural understanding, and communication along the international ways of thinking and seeing (Parmenter, 2000).

Commonly, internationalization at the national, sector, and institutional levels is defined as the process of integrating an international, intercultural, or global dimension, into the purpose, functions or delivery of higher education (Knight, 2004). Ellingboe (1998) added that internationalization is the ongoing process of integrating an international perspective into higher education institutions. It should encompass a multi-dimensional, inter-disciplinary, and future-oriented leadership vision, which involves the many stakeholders of the institution, in order to respond and adapt appropriately to the ever increasingly diverse and global environment. Hence, the many definitions and dimensions of internationalization have definitely given grounds to its complexity.

Ultimately, in the globalizing age, the world is getting smaller and no one wants to be left out, hence, in order to survive among the abrupt global changes. Higher education in Taiwan should face the difficult challenge of not only understanding both sides of the cultural values, but also diminishes the gap among different educational systems. 


\subsection{Campus Toastmasters club and its contributions}

Recent research with regards to campus Toastmasters clubs offers a proven and enjoyable way to practice communication and leadership skills. In addition, it matches the qualifications that are essential to the development of a speaking language such as having both a supportive atmosphere and a comfortable environment. In general, Toastmasters International is a non-profit organization that offers an educational program in which students can develop and enhance their public speaking and leadership skills (Sun, 2008). Founded in 1924 at Santa Ana, California, Toastmasters International has grown to become a world leader in helping people become more competent and comfortable in front of an audience. The nonprofit organization now has nearly 250,000 members in more than 12,500 clubs in 106 countries, offering a proven and enjoyable way to practice and develop one's communication and leadership skills (Toastmasters International, 2008b). Majority of these clubs, including those located in English as Foreign Language (EFL) regions, uses English as a communication language. Hence, the Toastmaster club serves two main purposes: it is a place in which students not only undergo public-speaking training but also practice their skills in English oral communication (Sun, 2008).

In Taiwan, the first Toastmasters club was established in 1958; the Taipei Toastmasters club, currently, the number has now grown to 146 clubs (Toastmasters International District 67, 2009a). Within these clubs a total of 112 are English clubs and with 20 of them situated within the higher education institutions in Taiwan as campus clubs or organizations (Toastmasters International District 67, 2009b). Campus Toastmasters works on ways to promote English language communication skills and enhance students' leadership capabilities beyond the four walls of the classroom. Most Toastmasters meetings are comprised of approximately 20 people who meet weekly (or once/twice a month in the case of Taiwan) for an hour or two. Members practice and learn skills by filling a meeting role, ranging from giving a prepared speech or an impromptu one to serving as timer, evaluator or grammarian. There is no instructor; instead, each speech and meeting is critiqued by a member in a positive manner, focusing on what was done right and what could be improved (Toastmasters International, 2008b). After undergoing a basic foundation training program; a series of 10 self-paced speaking assignments, members learn skills related to use of humor, gestures, eye contact, speech organization, and overall speech delivery (Toastmasters International, 2008a).

Besides communication skills, members also learn leadership skills by means of undergoing various carefully conceptualized training modules; a series of 10 projects in the Competent Leadership manual, and by serving the role of club officers. Members are also required to take on various active meeting roles and voluntarily serve as club's officers in district and/or division levels as well. Responsibilities are given to the members and opportunities to lead are provided (Toastmasters International, 2008a). Ultimately, the roles and agendas are designed based on an environment that is cooperative, supportive, and dynamic (Chiang, 2005). In essence, members learn by doing, which is also known as experiential learning (Dewey, 1937).

Beyond the regular Toastmasters club meetings, many researchers have adapted, modified, or integrated the Toastmasters way into actual classroom lessons (Chiang, 2001; Kuo \& Chen, 2009; Nordin \& Shaari, 2005; Sun, 2008). Chiang (2001) modified the Toastmasters speech training program and integrate it into a general conversation course program. This modified speech training program features low-threat conversation activities followed by a meeting-format speech-training program with multi-roles activity design through which each student is required to speak and interact with one another. Result shows that through role-taking, observation, and participation, student discovered the new roles they could perform well and modified their self-concept. In addition, advancement in their communication competence, which makes their public presentations more successful and enjoyable was clearly observed (Chiang, 2001).

Nordin and Shaari (2005) and Sun (2008) both reported the integration of the Toastmasters approach to their EFL learners. Both results show that such integration has enhanced the students' communication skills. Sun (2008) further added that the Toastmasters approach integrates various tasks into each meeting, hence, students 
reported improvement not only in their public-speaking skills but also in their English proficiency, their affective competence, and their social competence. Additional results concerning language skills progress such as listening, speaking, reading, writing, vocabulary, pronunciation, and communication, all indicated that the students considered the Toastmasters approach an effective facilitator for their English abilities (Sun, 2008).

College students nowadays are primarily involved in their studies and classroom activities; however, involvement in student clubs and organizations is also quite common. In a study involving the quality of student involvement in a group of college educational psychology students, findings suggest that overall quality of experience was greater during cooperative learning. Benefits occurred specifically for thinking on task, student engagement, perceptions of task importance, and optimal levels of challenge and skill (Peterson \& Miller, 2004). Similarly, in Taiwan, several studies involving college students have also pointed out that students learn more by becoming more involved (Chang, 1990; Huang \& Chang, 2004; Lin, 1990). In essence, for students' growth to take place, students need to actively engage in their environment. In a way, the effectiveness of any educational policy, practice, or program is directly related to the capacity of that policy, practice, or program to increase student involvement.

\section{Research methodology}

This study uses methodology from the qualitative paradigms in an attempt to enhance the strengths of data collection and advance students' insights surrounding the perceived contributions of campus Toastmasters club in the enhancement of the student members' global awareness. A qualitative research is basically concerned with an issue or problem where there are few or no earlier studies to refer to. The focus is on gaining insights and familiarity for later investigation. For data collection, the current study uses qualitative individual and focus group interviews. Kvale (1996) defined the qualitative research interviews as an attempt to understand the world from the subjects' point of view, to unfold the meaning of peoples' experiences, and to uncover their lived world prior to scientific explanations. Likewise, focus group interviews are among one of the most widely used qualitative research tools in social sciences studies. A probable benefit of this approach is that interviewees may feel greater confidence in a group setting, which may encourage them to offer comments and discuss matters they would not in a one-on-one interview (Axinn \& Pearce, 2006).

Participants of the qualitative focus group interview are volunteer officers and members coming from the different 20 campus Toastmasters clubs all over Taiwan. The volunteer sampling method was used in the selection of participants. A volunteer sample is a sample consisting of units of the population that chose to respond (Zuo, 2006). Since, the current study is about enhancing the students' global awareness, campus Toastmasters club members that are willing to contribute and participate are deemed more effective source of information. In order to organize the focus group sessions, an invitation was send to all campus Toastmasters clubs stating the objective of the meeting and possible dates the members are willing to participate. Five focus group sessions with a total of 60 campus Toastmasters members coming from 10 different schools were interviewed. Generally, each campus Toastmasters club comprises of at least 20 student members. Therefore, 60 students approximately represent $15 \%$ of the total 400 student members all over Taiwan.

A total of $40(67 \%)$ officers and $20(33 \%)$ campus Toastmasters club members participated in the sessions. Follow-up individual interviews were also accomplished to further clarify some difficult issues gathered from the preliminary focus groups sessions. Similar questions regarding on how to enhance college students' global perspective and increase its diversity on its membership in joining the campus Toastmasters club were asked in all sessions. Interview data were then transcribed and analyzed using the Miles and Huberman (1994) method for generating meaning. Table 1, shows the background demography of the participants. Participants' gender shows that there is a slight higher participation of female than male student members (Female 53\% or 32 members and Male $47 \%$ or 28 members) in the focus group sessions. In addition, as mentioned earlier the number of officers comprises of almost two thirds of the total participants with their corresponding roles and ranks provided in the table 1. Average age of the participants is around 21 years old, while the average membership is around 16.5 
Hsu, T. C.

months. Background demography suggests that participants are quite knowledgeable with the topics in questions, since they have long been involved in organizing the campus Toastmasters club. Further insights into the research questions shall be provided in the following section.

\section{Table 1}

Participants demography $(N=60)$

\begin{tabular}{|c|c|c|c|c|}
\hline Item & $n$ & $\%$ & Mean & SD \\
\hline \multicolumn{5}{|l|}{ Gender } \\
\hline Female & 32 & 53 & & \\
\hline Male & 28 & 47 & & \\
\hline \multicolumn{5}{|l|}{ Positions } \\
\hline President & 5 & 13 & & \\
\hline Vice-President (Education) & 5 & 13 & & \\
\hline Asst. Vice-President (Education) & 5 & 13 & & \\
\hline Vice-President (Membership) & 2 & 5 & & \\
\hline Asst. Vice-President (Membership) & 3 & 8 & & \\
\hline Vice-President (Public Relations) & 4 & 10 & & \\
\hline Asst. Vice-President (Public Relations) & 1 & 3 & & \\
\hline Secretary & 3 & 8 & & \\
\hline Treasurer & 2 & 5 & & \\
\hline Past officers & 8 & 21 & & \\
\hline Age (years old) & & & 21 & 3 \\
\hline 18 & 5 & 8 & & \\
\hline $19-20$ & 21 & 35 & & \\
\hline $21-22$ & 8 & 13 & & \\
\hline $23-24$ & 5 & 8 & & \\
\hline $25-26$ & 5 & 8 & & \\
\hline $27-28$ & 2 & 3 & & \\
\hline $29-30$ & 0 & 0 & & \\
\hline 31 & 1 & 2 & & \\
\hline Missing & 13 & 22 & & \\
\hline Membership (months) & & & 16.50 & 15.50 \\
\hline $1-6$ & 18 & 30 & & \\
\hline $7-12$ & 20 & 33 & & \\
\hline $13-18$ & 7 & 12 & & \\
\hline $19-24$ & 1 & 2 & & \\
\hline $25-30$ & 6 & 10 & & \\
\hline $31-36$ & 5 & 8 & & \\
\hline More than 36 months & 3 & 5 & & \\
\hline
\end{tabular}

\section{Results and discussions}

The primary objective of this study is to examine how to increase college students' global awareness through participation in the campus Toastmasters club. Results shall be separated into two sections by means of the research questions.

\subsection{How can Toastmasters enhance college students' global perspective?}

In the campus Toastmasters club, student members undertake different speaking assignments and various active meeting roles. During the focus group interview, when the students were asked on how to improve their club so that their members can gain more global viewpoints. Students mentioned that the provision of different group activities can indeed help students create and developed their global perspective. This is actually one of the major components of the campus Toastmasters club, which is to have a variety of cooperative learning 
activities that are geared towards language and character improvements. Initially, students primarily joins Toastmasters club because of the notion of improving their language and meeting new friends. However, this can be minimized after the students realized the true objectives of the club. In essence, campus Toastmasters are not only language clubs, but school organizations that helped students gain global awareness and perspectives. Some responses during the focus sessions regarding how to enhance students' global perspectives are as follows:

In order to enhanced our global views. During the meeting, we can actually focus on something globally popular, such as the World cup soccer games, NBAs or Baseball. The whole world is watching such games. I'm sure we can have lots of topics. (FGT 1, Club member's response)

Another person echoed the above student's comment as follows:

We can also form a study group. We can invite them (international students) and share different views on global issues. It's really a kind of interaction and sharing of ideas. (FGT4, Club member's response)

Both of these quotations have suggested that related activities outside of the normal meetings could also enhance the learners' global views. Both different club members found out that such activities could benefit their global perceptions. Similar response is then followed by another club officer's answer with regards to his perception of enhancing global views as follows:

We can widen our global perspective when we work as a volunteer, we can go to different countries and serves as a volunteer. We can go places and exchange cultural experiences. (FGT 5 , Club officer's response)

FGT5 echoed the effectiveness of working as a volunteer or going to different countries as a volunteer. In doing so, it implied that a successful outcome can enhanced the student members' global viewpoints through exchanges in working project and cultural experiences. Furthermore, the following student officer; FGT 6 suggested a good example of creating global perceptive at the same time attracting newcomers' attention:

Creating a common interest within international issues can also help enhance the global perspectives for newcomers. For example, we can talk about the latest fashion or the latest foreign singer, such topics that appeals to the members will surely make the meetings interesting. (FGT 6, Club officer's response)

Another good example also related to the same concept:

We can actually invite students from other colleges, or international faculty or professors, and we can also invite our senior members or international student members to join our meetings... and the fun thing is that we can also have movie or film showing events, discussions, and even book readings in our club. (FGT 7, Club officer's response)

Results indicate that the above two different club officers tend to create a common interest regarding international issues. More so, the opening up of opportunity with regards to enhancing the students' global views through friendly and non-hostile strategies is definitely helpful. More importantly, campus Toastmasters can utilize their membership not only limited to the campuses or universities all over Taiwan, but also professionals in all other fields of industries. Such resources can undoubtedly make meetings more fruitful and interesting, in essence, opens a global window for the students to see and experience.

Additional insights were also noted:

The conclusion (is that) how we can improve our global perspective, we can try to create different themes in our meetings, such as international themes and globalization. We can also invite foreign students and alumni students like John (a former officer/member, who had the 
experience of studying abroad). He has experiences to work and study in another country. (FGT

2, Club officer's response)

So, we think that knowing the cultural differences and reaching a point of familiarity is the most important aspect in English speaking. That is actually the reason why we learn English. We believed that having a global point of view is the ultimate achievement of being united as a global citizen. (FGT6, Club officer's response)

In order to improve student's global views and reach a point of familiarity; FGT 2 and 6 considered choosing themes such as concerning more on international issues and/or globalization. In order to diminish the differences between two sides of communication, English language is the vehicle for college students to bridge the gap amongst international students and local students. More so, the club officer; FGT6, mentioned being united and becoming a global citizen as the ultimate goal of joining the campus Toastmasters club, which is actually quite an important point of view.

As per findings from the above sections, most informants tend to know the differences and reach the familiarity towards global views while being a campus Toastmasters member. Besides improving on their English language communication skills, most club officers present in the focus group sessions suggested that the provision of the various cooperative learning strategies such as structured interaction and communication activities, and individual speaking activities, while not worrying about their grammatical errors. Is a means of sharing various cultural concepts within the conducted Toastmasters meeting program, hence, increased their global awareness.

\subsection{How can Toastmasters increase its diversity on its membership in order to gain more global point of views?}

In the campus Toastmasters club, student members undertake different speaking assignments and rotate different active meeting roles. During the focus group interview, when the students were asked how to increase Toastmasters diversity so that student members would gain more global viewpoints. The following section explained the reasons why campus Toastmasters members would expect more international students to join the campus Toastmasters club:

We feel that having international members is very important. If international members can give us better evaluation (speech), they can use different words and give us comments and examples of different cultural aspects of communications. The second is we can make different friends and learn to exchange our cultural experiences. We can know something new in their countries. And we can know different words related to their culture. (FGT 3, Club officer's response)

The key phrase here is that "We feel that having international members is very important". Once there is certain number of international students joining Toastmasters club, local student members would find the gaining of different global opinions easily. Furthermore, FGT3 also stated another crucial phrase is that "we can make different friends and learn to exchange our cultural experiences". In so doing, students would gain cultural experiences through positive relationship among members in-and-out of club meeting. In addition, during the meeting sessions, international members tend to provide (in the meeting) a better evaluation (whether speech or language). They can use different words (words that are seldom used in Taiwan) and give different comments and cultural aspects of communications, which would benefit other members' different point of views in terms of their language and cultural experiences.

When focusing more on the ways and means to increase Toastmasters members' diversity on its membership, in order to help members gain a greater sense of global viewpoint and the strong expectations of creating a more English speaking environment. A student mentioned that,

There are actually several points we can do: First, we can coordinate with the schools' foreign 
language center, so we can recruit more international students to come and join the Toastmasters. Second, we can invite some foreigners (teachers or some well-known individuals) as speakers in the meetings, which can attract international students. Third, we need to conduct more high quality meetings because it is only this way that we can create a better English environment and lastly, let's have more champions, what I mean is let our members join competitions. (FGT6, Club officer's response)

The first strategy is to recruit international student members in the foreign language center (or any other areas where international students frequently stays), which are interested in having language exchanges. FGT6 finds it helpful with respect to the needs of language exchange experiences, and by doing so will also help attract more international student members. In addition, the strategy of inviting foreign speakers to speak in Toastmasters meetings is also a great idea (which is actually a re-occurring strategy that most campus Toastmasters uses). Furthermore, FGT6 would think that creating more English environment in Toastmasters meetings with high quality meeting roles and meetings activities would not only make the meetings enjoyable and productive, but it also a channel in bettering our local speakers and help create future competition champions.

Another respondent; FGT1 had somewhat similar notion as follows:

Joining the language exchange programs is actually a great way to attract international student members. (FGT1, Club member's response)

This statement suggests that this strategy would not only aid our local students in learning (or crossing) the cross-cultural barriers, but simultaneously learns and practices a foreign language. More importantly, is the concept of having a new friend; students can gradually introduce and invite the international students in the meetings. With the Toastmasters being an international organization, the international student might actually be already familiar with the organization, hence, make recruitment easier.

Another respondent had a quite different approach with the use of technology to the issue:

We can actually utilize the internet and post some 'you tube' videos on the campus Toastmasters Website, because I think that the Website is the most direct place for people to know your club, therefore, we can post our famous speech videos on the Website and show what students can achieve in our club. (FGT8, Club member's response)

This third strategy mentioned by FGT8 has also been proven to attract not only local Toastmasters membership, but has also drawn the attention of international student members. The respondent further stressed that he believed in doing so, meeting activities would be clearer, as the saying goes 'videos (or pictures) is worth more than a thousand words' in itself is a great way to advertise and promote the essence of a campus Toastmasters club.

Another respondent; FGT4 presented another outlook on how to attract both local and international membership:

In order to attract local and international membership, what we can do is first to recruit international student members, then looking for the local members would be easy. A main idea is that international students come to Taiwan with the idea of making cultural exchanges, learn more about Taiwan, and have new friends, hence, I believe that as we share to international students the goal of our club, they will readily join us. As the local students see that campus Toastmasters is international (membership), they will come and join us. (FGT4, Club officer's response)

As indicated above, FGT 4 reflected his ideas on how to increase Toastmasters diversity on its membership 
Hsu, T. C.

on both side of the spectrum, both the local and international students joining Toastmasters meetings. Up to this point, he used a quite different concept to attract the Toastmasters diversity on its membership. For example, he stated that he would provide more opportunity for international student members to have cultural exchanges, learns more about Taiwan, and meet new friends in and outside campus. At the same time, such activities will no doubt attract the local students' attention and eventually will want to join the club.

In the next sample, respondent pointed out some new perspective related to information technology on how to recruit more international student members:

The main point to push is innovation. What actually have more to offer than Toastmasters meetings and events, I think we also did a lot of things outside of the normal Toastmasters meeting. We can use the school's bulletin board services (or BBS), to promote what we are doing. It's been there for awhile now and it's quite a great way to communicate with the student population. So if it works for the other clubs, it would also work for us. Try it. (FGT 1, Club officer's response)

When Toastmasters students take on club responsibilities, they are obliged to keep the meeting sessions running smoothly. In most cases, club officers tend to recruit more members to join the club. Currently, most campus Toastmasters clubs builds on the concept of attracting more diversity on its membership in order to increase student members' global viewpoints. FGT1; a club officer, simply thought of taking the advantage of using information technology such as the school's BBS to advertise Toastmasters meetings, events, and activities.

Another respondent reflected on his global perspective with respect to the result of gaining more diversity on its membership in Toastmasters. He said:

We are an international club and since the world is getting a lot smaller each day. Understanding both sides (East and the West) and knows what the other wants. You must first learn to understand what they want and give it to them; and then know what you want from them and get that from them. That'll be a perfect world if you can understand: what to give and what to get. (FGT4, Club officer's response)

Another respondent had similar ideas of recruiting international members:

We have to understand people. The more we understand what people want; the more we will give what they want and get what we want from them. That's a very business philosophy. Understand what students want and you will know how to get your members. (FGT8, Club officer's response)

During the focus group interview sessions, when the students were asked what the perceived global view in joining the campus Toastmasters club, FGT4 stated that 'the world is getting a lot smaller'. Understanding both sides (denotes both the East and West) and giving to get what you want. With regards to the issue of recruiting new members, such phenomenon mostly happens to every established campus club. With the student officers still new and lacks experience in handing this issue, new members tends to shy away gradually.

In order to minimize such issues on how to recruit new members every now and then, understanding people is an important issue to further improve the joining numbers during the Toastmasters meeting. For instance, another Club officer; FGT8 stated that understanding what students wants and you will tell Toastmasters in order to get the needed members that you will influence your club for the better. Students mentioned that since we are an international clubs, and joining the campus Toastmasters club is to create a better world if one can understand: what to give and what to get. The objective is to diminish the differences among Toastmasters members, and further understand each other taking the first step in creating a better world.

Additional, response includes: 
... if you don't have the chance to go outside Taiwan and see the world for yourself. They (international students) came to Taiwan for a reason; they want to know more about Taiwan. So they choose Taiwan... So that's make Toastmasters more international. (FGT5, Club officer's response)

The above student; FGT 5 stated that his world viewpoint (perspective) is to make campus club more international because he believes that international students come to Taiwan for some reasons. This statement suggested that international students would be more willing to get involve in Toastmasters because Toastmasters members' diversity on its membership would attract other international students in joining Toastmasters.

Furthermore, these international student members would gain a greater sense of global viewpoints with strong expectations to know Taiwan better. In so doing, local members would gain a greater sense of global viewpoints with strong expectations to create a more English speaking environment and communicate with other international students within the campus environment. By implication, the belief that international students choose to study in Taiwan's colleges and academic institutes for certain reasons and one of such reasons is to get to know Taiwan better, opportunities to have international members is therefore not a difficult undertaking.

In sum, as Taiwan's higher education institutions becomes more internationalize, the notion of adapting the Toastmasters international approach in organizing the campus Toastmasters clubs throughout Taiwan; wherein memberships are voluntary, is of a new and creative approach. In terms of promoting the global views, enhancing college students' global perspective, and attract more international student members on its diverse membership are some strategies that are adopted to reinforce the local students' global views and ultimately help diminished their cultural shock.

\section{Conclusion}

Prior to the beginning of this study, the researcher had investigated many related issues with regards to the current college Toastmasters members' perception in terms of the trend of globalization and internationalization. From the actual data gathered during the focus group interviews, results have indeed helps reinforced the concerns with respect to the co-curricular activities in Taiwan's university education influenced by the rapidly changing world, and that neither one can ignore its impetus and implication. In addition, within the concept of cooperative learning, promotion of global views by means of sharing various cultural concepts within the conducted Toastmasters meeting program, group works such as structured interaction and communication activities, and individual speaking activities, while not worrying about their grammatical errors are also used as strategies. To summarize, current campus Toastmasters club members among the twenty academic institutes in Taiwan's higher education milieu would tend to get more opportunities in obtaining a global perception through the opportunity of meeting more international students in their social environment.

\subsection{Implications}

Several key implications derived from these findings may further aid future researchers and school administrators alike:

D Understand what college Toastmasters members need; moreover, bring more international students and help them meet their needs as well.

$>$ Increase college Toastmasters members' different global views and help identify its similarities and differences within the different cultural concepts, especially helping both the international and local students bridge their cultural differences.

> Help more local college Toastmasters members know their identity, and help build up their confidences in using effective communication and leadership skills through Toastmasters training and public speaking events. 
Hsu, T. C.

\subsection{Recommendations and Suggestions}

The above evidence from the data demonstrates that the college Toastmasters members' attitudes regarding issues on global perspective. There appeared to be a likely positive relationship created in Toastmasters meeting between local and international students while utilizing many excellent ways in recruiting many different international students to join Toastmasters membership. While enhancing college students' global perspective and attracting more international students on its diverse membership would be the focus on this study. However, the researcher observed that there are no objective evidences on how to measure the Toastmasters members' achievement in terms of helping them increase their global perspective. In the following are some recommendations for future researchers and/or Toastmasters advisors:

$>$ Create a global perspective in a conducted Toastmasters meeting by utilizing a large number of international students.

$>$ Create an innovative global perspective other than the normal meeting concept.

$>$ Diminish the barriers among different themes discussions or cultural issues once members show concerns or questions.

$>$ Invite more college faculty and staff in campus Toastmasters meeting so that both the students and teachers can generate a two-way communication platform in terms of reinforcing global perspective in higher education milieu.

Nevertheless, while Taiwanese higher education institutions are focusing on the development towards global competitiveness, this study will be able to inform school administrators or/ and professors on ways to help more university students promote global views and enhance students' capabilities beyond the four walls of the classroom. 


\section{References:}

Axinn, W. G., \& Pearce, L. D. (2006). Mixed method data collection strategies. New York: Cambridge University Press. <http://dx.doi.org/10.1017/CBO9780511617898>

Bohm, A., Meares, D., \& Pearce, D. (2002). Global student mobility 2025: Forecast of the global demand for international higher education. Sydney: IDP Education.

Chang, S. M. (1990). The impacts of colleges on students [In Chinese]. Taipei, Taiwan: Teacher Chang Culture Press.

Chiang, F. (2001). Implementing the Toastmaster tradition in the university language classroom setting Hwa Kang Journal of TEFL, 7, 99-116.

Chiang, F. (2005). Toasting our learning: Introducing Yang Ming university Toastmasters club. Journal of General Education, 8, 147-161.

Chin, J. M.-C., \& Ching, G. S. (2009). Trends and indicators of Taiwan's higher education internationalization. The Asia-Pacific Education Researcher, 18(2), 185-203. doi:10.3860/taper.v18i2.1322 $<$ http://dx.doi.org/10.3860/taper.v18i2.1322>

Coldstream, P. (1997). Training minds for tomorrow's world: On independence, curiosity, and effectiveness. Higher Education in Europe, 22(4), 507-515. doi:10.1080/0379772970220407 $<$ http://dx.doi.org/10.1080/0379772970220407>

Dewey, J. (1937). Experience and Education. New York: Simon and Schuster.

Ellingboe, B. J. (1998). Divisional strategies to internationalize a campus portrait: Results, resistance, and recommendations from a case study at a US university. In J. A. Mestenhauser \& B. J. Ellingboe (Eds.), Reforming the higher education curriculum: Internationalizing the campus (pp. 198-228). Washington, DC: American Council on Education.

Grunzweig, W., \& Rinehart, N. (Eds.). (2002). Rocking in red square: Critical approaches to international education in the age of cyberculture. Berlin: Lit Verlag.

Huang, Y. R., \& Chang, S. M. (2004). Academic and cocurricular involvement: Their relationship and the best combinations for student growth. Journal of College Student Development, 45(4), 391-406. doi:10.1353/csd.2004.0049 <http://dx.doi.org/10.1353/csd.2004.0049>

Knight, J. (2004). Internationalization remodeled: Definition, approaches, and rationales. Journal of Studies in International Education, 8(1), 5-31. doi:10.1177/1028315303260832 $<$ http://dx.doi.org/10.1177/1028315303260832>

Ko, S. L. (2008). International students in Taiwan at an all-time high. Retrieved June 11, 2008, from http://www.taipeitimes.com/News/taiwan/archives/2008/02/12/2003400913

Kuo, L. C., \& Chen, J. W. (2009). Integrate Toastmasters program into junior high school English classroom to enhance students' speaking ability. Paper presented at the Fourth International Conference on English Education and Drama.

Kvale, S. (1996). Inter views: An introduction to qualitative research interviewing. Thousand Oaks, CA: Sage.

Lin, Y. F. (1990). The relationship among academic involvement, learning patterns, and academic outcomes [In Chinese]. The Journal of Counselling, 13, 79-128.

Miles, M., \& Huberman, M. (1994). Qualitative data analysis (2nd ed.). Beverly Hills, CA: Sage.

Noels, K. A., Clément, R., \& Pelletier, L. G. (1999). Perceptions of teachers' communicative style and students' intrinsic and extrinsic motivation. The Modern Language Journal, 83(1), 23-34. doi:10.1111/0026-7902.00003 < http://dx.doi.org/10.1111/0026-7902.00003>

Nordin, S. M., \& Shaari, Z. H. (2005). Implementing the Toastmasters tradition in the L2 classroom. The English Teacher, 34, 60-66.

OECD. (2006). Education at a glance: OECD indicators 2006. Paris: Organisation for Economic Co-operation and Development.

Olsen, A. (2003). E-Learning in Asia: Supply and demand. Retrieved September 25, 2008, from http://www.bc.edu/bc_org/avp/soe/cihe/newsletter/News30/text004.htm

Parmenter, L. (2000). Internationalisation. In M. Byram (Ed.), Routledge encyclopedia of language teaching and learning. New York: Routledge.

Peterson, S. E., \& Miller, J. A. (2004). Comparing the quality of students' experiences during cooperative learning and large-group instruction. The Journal of Educational Research, 97(3), 123-134. doi:10.3200/JOER.97.3.123-134 <http://dx.doi.org/10.3200/JOER.97.3.123-134>

Scott, P. (1998). Massification, internationalization and globalization. In P. Scott (Ed.), The globalization of 
Hsu, T. C.

higher education. Buckingham: SRHE and Open University Press.

Stiglitz, J. E. (2002). Globalization and its discontents. New York: W. W. Norton \& company.

Sun, Y.-C. (2008). The Toastmasters approach: An innovative way to teach public speaking to EFL learners in Taiwan. RELC Journal, 39(1), 113-130. doi:10.1177/0033688208091143 $<$ http://dx.doi.org/10.1177/0033688208091143>

Toastmasters International. (2008a). How does it work? Retrieved January 11, 2010, from http://www.toastmasters.org/MainMenuCategories/WhatisToastmasters/HowDoesItWork.aspx

Toastmasters International. (2008b). What is Toastmasters? Retrieved January 11, 2010, from http://www.toastmasters.org/MainMenuCategories/WhatisToastmasters.aspx

Toastmasters International District 67. (2009a). Dig more, reap more. Retrieved December 27, 2009, from http://www.toastmasters.org.tw/

Toastmasters International District 67. (2009b). English club. Retrieved December 27, 2009, from http://www.toastmasters.org.tw/d67web/en/clubs/club-english.htm

Zuo, J. (2006). Elements of statistics. Retrieved March 5, 2011, from

http://www.ams.sunysb.edu/ jasonzou/ams102/notes/notes7.pdf 